\title{
Wir brauchen lebendige Träume und Selbstachtsamkeit
}

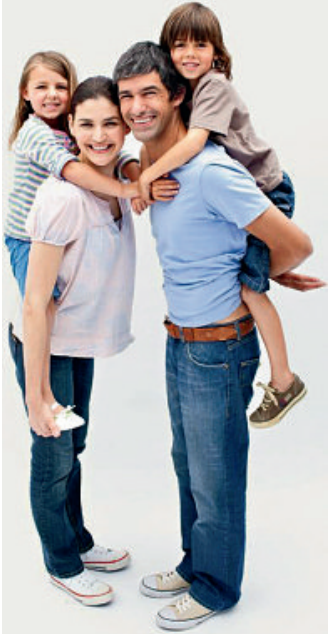

Für ein erfülltes und erfolgreiches berufliches Leben sind Freunde und Familie wichtig - und Selbstachtsamkeit.

1 Bei dieser Gelegenheit weise ich gerne auf das von der FMH initiierte Programm ReMed hin, in dem Kolleginnen und Kollegen Ärzte in schwierigen persönlichen oder beruflichen Situationen unterstützen (Hersperger M. ReMed - Unterstützung zum richtigen Zeitpunkt. Schweiz Ärztezeitung. 2010;91(44):1717

2 Resnick MD, Bowles G. Us and them: Worldwide health issues for adolescents. Lancet 2007; 369(9567):1058-60. Resnick MD. Best bets for improving the odds for optimum youth development. In: Kline KK (ed.). Authoritative Communities: The Scientific Case for Nurturing the Whole Child. New York: Springer; 2008. S. 137-150.
Das Institut universitaire de médecine sociale et préventive in Lausanne, heute unter der Leitung von Prof. F. Paccaud, dem Nachfolger von Prof. F. Gutzwiller, feierte im Januar 2011 sein 40-jähriges Bestehen. Die Gründung des Instituts durch Prof. Armand Delachaux fiel damit in eine Zeit, in der die Disziplin der «Public Health» erst in unsere Fakultäten Einzug hielt. Gelegenheit für einen Rückblick, wie sich das IUMSP im Laufe der Jahre zu einem Ort der Wissenschaft von internationalem Rang entwickelt und unter anderem wichtige Brücken zwischen der «Volksgesundheit/-medizin» und der klinischen Forschung geschlagen hat: sei es auf dem Gebiet der klinischen Epidemiologie, der Herz-Kreislauf-Erkrankungen, durch ein Krebsregister sowie durch Forschung auf dem Gebiet der Gesundheitsdienste usw. oder auch durch die Unité multidisciplinaire de santé des adolescents, UMSA, der Prof. P.-A. Michaud vorsteht.

Ein keynote speaker war Michael D. Resnick, Spezialist für Jugendmedizin der Universität Minnesota. Eine Überraschung: Er hielt seinen von sozialer Ethik und Spiritualität geprägten, hochinteressanten Vortrag streng ex cathedra - ohne PowerPoint ... Er sprach über das, was Jugendliche brauchen, um zu kompetenten und ausgeglichenen Erwachsenen heranzureifen, und betonte, dass in der heutigen komplexen Realität die Gesundheit der Jugendlichen nicht mehr allein in der Verantwortung der Familie liegen könne - eine in konservativen Kreisen verpönte Ansicht. Schule und andere Kreise müssten ihren Beitrag leisten. Eine Veränderung der letzten 15 oder 20 Jahre betreffe die Tatsache, dass die Jugendlichen die Erwachsenen - einschliesslich der Lehrkräfte an den Hochschulen - nicht mehr als erste Informationsquelle benötigten. Unsere Rolle bestehe vielmehr in der Interpretation, so Resnick, damit wir der jungen Generation helfen zu verstehen, welche Information von Wert ist. Im Internet finde man korrekte und falsche Informationen, und wir müssten deutlich darauf hinweisen, was auf soliden Grundlagen basiere und was nicht.

Wir müssen «uns unsere Träume erhalten - ein Thema, das nur selten in Tagungen wie dieser angesprochen wird. Wenn wir uns an Dingen nicht mehr freuen, sie nicht mehr geniessen können, fällt es schwer, uns für die Heilung einer beschädigten Welt einzusetzen, die aber unsere Hände und unser Herz braucht.» Dabei gehe es in erster Linie darum, Freundschaft und Familienleben wertzuschätzen: «Ich hoffe, dass jeder von uns eine gute Mutter, ein guter Vater und ein verlässlicher Freund ist.» Man muss dazu stehen, dass die auf das eigene Ich gerichtete Achtsam- keit kein Egoismus ist, sondern die Quelle der Kraft für wichtige Kämpfe. Man darf nicht glauben, dass man dessen nicht fähig ist! Eleanor Roosevelt sagte, niemand könne uns zwingen, uns minderwertig zu fühlen, wenn wir dies nicht selbst zuliessen.

Wie soll man es aber anstellen, dass man sich nicht doch überfordert fühlt? «Man muss filtern, entscheiden können zwischen dem, was wir an uns heranlassen, und dem, was wir ablehnen, weil es uns selbst oder unsere Arbeit in Gefahr bringt. Daher dürfen wir nur akzeptieren, womit wir auch umgehen können.»

Und darüber hinaus? In sich hineinblicken, mindestens zweimal täglich innehalten und tief einatmen. Und ich denke an dieser Stelle an den Appell der beeindruckenden Ingrid Betancourt Mitte Februar in Lausanne, die sagt, wir dürften nicht immer wieder gute Gründe finden für unser Unglücklichsein.

«Sich auf Freunde stützen können, einen ruhigen Ort in der Natur finden und das Gefühl der Dankbarkeit, der Ehrfurcht, des ‘Wunderns〉 und des Mysteriums wieder einmal zulassen.» Selbst wenn dringende Fragen unsere Tatkraft und unsere geistigen Kapazitäten brauchen, müssen wir uns die Zeit nehmen und an uns selbst arbeiten. «Erinnern Sie sich daran, wie schön Sie sind, wenn Sie lächeln. Pflegen Sie Ihren Körper. Denn wenn Sie dies tun, spüren die anderen Ihre Energie, wie ein Radio mit einem guten Empfang, der Lärm in Musik verwandelt (...). Zweifeln Sie nie daran, dass Ihr Tun einen Fortschritt bringen wird.»

Trotz des amerikanischen Enthusiasmus, den man zuweilen als übertrieben empfinden kann, hat M. Resnick wichtige Botschaften vermittelt: Unsere Rolle gegenüber der jungen Generation hat sich verändert. Unser eigenes Wissen begründet objektiv betrachtet nicht mehr unsere Überlegenheit. Dennoch (dies ist meine persönliche Meinung) können wir uns damit trösten, dass sich Erfahrung weder in Büchern noch im Internet erwerben lässt (bis zu dem Zeitpunkt, an dem es der Fortschritt auf dem Gebiet der Neurowissenschaften ermöglicht, diese Erfahrung von einem auf den anderen passiv zu transponieren). Unsere Rolle als Lehrer oder Tutor muss im Zuhören, im «Coaching», in der Super- und Intervision und im Austausch bestehen. Und darin, dass wir uns um uns selbst kümmern. Denn dies ist Prävention gegen Burn-out oder Erschöpfungssyndrome. Damit wir unsere Freude an der Arbeit erhalten - und zuweilen auch daran, nicht zu arbeiten $[1,2]$.

Jean Martin, Mitglied der Nationalen Ethikkommission und der Redaktion 